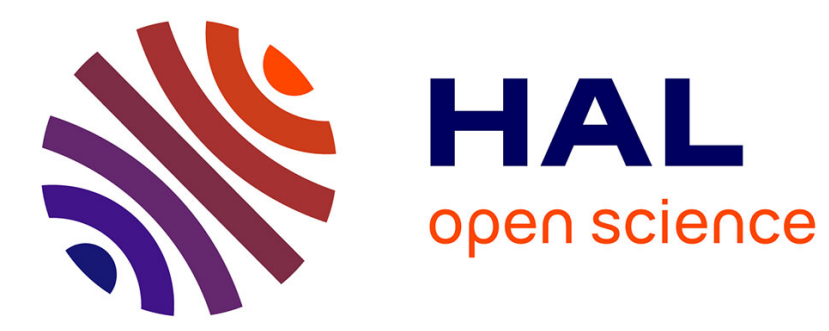

\title{
Spectral interference of terahertz pulses from two laser filaments in air
}

Yanping Chen, Zhelin Zhang, Zhen Zhang, Xiaohui Yuan, Feng Liu, Min

Chen, Jianqiu Xu, Jin Yu, Zhengming Sheng, Jie Zhang

\section{- To cite this version:}

Yanping Chen, Zhelin Zhang, Zhen Zhang, Xiaohui Yuan, Feng Liu, et al.. Spectral interference of terahertz pulses from two laser filaments in air. Applied Physics Letters, 2015, 106, pp.221105. 10.1063/1.4922143 . hal-02308171

\section{HAL Id: hal-02308171 \\ https://univ-lyon1.hal.science/hal-02308171}

Submitted on 4 Feb 2021

HAL is a multi-disciplinary open access archive for the deposit and dissemination of scientific research documents, whether they are published or not. The documents may come from teaching and research institutions in France or abroad, or from public or private research centers.
L'archive ouverte pluridisciplinaire $\mathbf{H A L}$, est destinée au dépôt et à la diffusion de documents scientifiques de niveau recherche, publiés ou non, émanant des établissements d'enseignement et de recherche français ou étrangers, des laboratoires publics ou privés. 


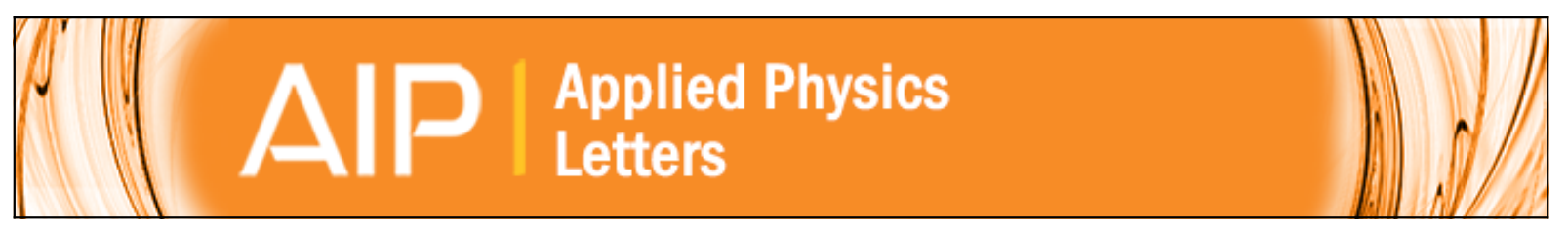

\section{Spectral interference of terahertz pulses from two laser filaments in air}

Yanping Chen, Zhelin Zhang, Zhen Zhang, Xiaohui Yuan, Feng Liu, Min Chen, Jianqiu Xu, Jin Yu, Zhengming

Sheng, and Jie Zhang

Citation: Applied Physics Letters 106, 221105 (2015); doi: 10.1063/1.4922143

View online: http://dx.doi.org/10.1063/1.4922143

View Table of Contents: http://scitation.aip.org/content/aip/journal/apl/106/22?ver=pdfcov

Published by the AIP Publishing

\section{Articles you may be interested in}

High-field half-cycle terahertz radiation from relativistic laser interaction with thin solid targets

Appl. Phys. Lett. 103, 204107 (2013); 10.1063/1.4831684

Scaling and saturation of high-power terahertz radiation generation in two-color laser filamentation Appl. Phys. Lett. 102, 201113 (2013); 10.1063/1.4807790

Longitudinal terahertz wave generation from an air plasma filament induced by a femtosecond laser Appl. Phys. Lett. 102, 151106 (2013); 10.1063/1.4802482

Experimental observation of a traveling plasma grating formed by two crossing filaments in gases

Appl. Phys. Lett. 98, 121110 (2011); 10.1063/1.3568888

Interference fringes from stabilized diode lasers

Am. J. Phys. 68, 245 (2000); 10.1119/1.19416

Frustrated by

old technology?

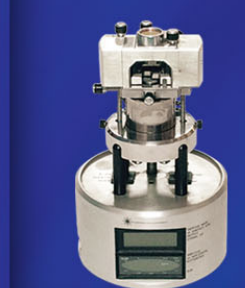

Is your AFM dead

and can't be repaired?

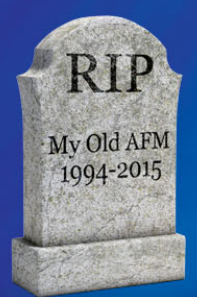

Sick of bad customer support?

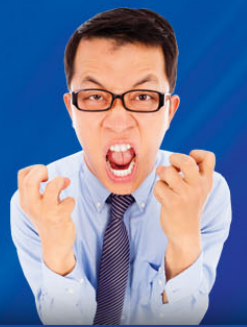

It is time to upgrade your AFM

Minimum $\$ 20,000$ trade-in discount for purchases before August 31st

Asylum Research is today's technology leader in AFM 


\title{
Spectral interference of terahertz pulses from two laser filaments in air
}

\author{
Yanping Chen, ${ }^{1,2, a)}$ Zhelin Zhang, ${ }^{1,2}$ Zhen Zhang, ${ }^{1,2}$ Xiaohui Yuan, ${ }^{1,2}$ Feng Liu, ${ }^{1,2}$ \\ Min Chen, ${ }^{1,2}$ Jianqiu Xu, ${ }^{1,2}$ Jin Yu, ${ }^{1,3}$ Zhengming Sheng, ${ }^{1,2,4, b)}$ and Jie Zhang ${ }^{1,2}$ \\ ${ }^{1}$ Key Laboratory for Laser Plasmas (Ministry of Education), Department of Physics and Astronomy, \\ Shanghai Jiao Tong University, Shanghai 200240, China \\ ${ }^{2}$ IFSA Collaborative Innovation Center, Shanghai Jiao Tong University, Shanghai 200240, China \\ ${ }^{3}$ Institut Lumière Matière, UMR 5306 Université Lyon 1-CNRS, Université de Lyon, \\ 69622 Villeurbanne Cedex, France \\ ${ }^{4}$ Department of Physics, SUPA, University of Strathclyde, Glasgow G4 ONG, United Kingdom
}

(Received 12 February 2015; accepted 23 May 2015; published online 3 June 2015)

\begin{abstract}
Spectral interference is experimentally demonstrated by two terahertz pulses emitting from filaments induced by two successive femtosecond laser pulses in air. Here, a leading pulse is set to be weaker than a trailing pulse and their temporal separation is larger than the pulse duration of the terahertz pulses. When the leading pulse is stronger than the trailing pulse, the frequency modulation within the whole terahertz envelope is greatly deteriorated due to nonlinear effects applying on the trailing pulse through the plasmas generated by the leading pulses. Such unique terahertz spectrum may find applications in terahertz spectroscopy. (C) 2015 AIP Publishing LLC.

[http://dx.doi.org/10.1063/1.4922143]
\end{abstract}

The technique of terahertz time-domain spectroscopy (THz-TDS) emerges to become valuable for potential applications in many areas such as security, communication, and medicine. For example, in the security area, not only can $\mathrm{THz}$ waves propagate through cloths and packaging materials but also they can be used to identify immediately most of the hidden explosives, which feature unique "fingerprints" within terahertz region. ${ }^{1-4}$ Laser-induced plasmas, photoconductive terahertz antennas, and nonlinear crystals are effective terahertz emitters to generate broadband terahertz pulses which can be used for terahertz time-domain spectroscopy. ${ }^{5-8}$ In some occasions, however, these terahertz sources are not powerful enough to provide reliable spectra, especially in the case that the THz-TDS system is working in a reflective mode. The normal way to obtain a more reliable terahertz spectrum is to increase the data-acquisition time.

In this letter, we demonstrate spectral interference of two terahertz pulses emitting from femtosecond laser filaments with temporal separation larger than the pulse duration of the terahertz pulses. The terahertz spectral signals are enhanced by the interference of two broadband terahertz pulses, which could benefit the resolution of the spectrum for a THz-TDS system.

In our experiments, a $1 \mathrm{kHz}, 800 \mathrm{~nm}, 40 \mathrm{fs}$ Ti-sapphire laser beam was split into two (a reference beam and a control beam). The energy of the reference and control beams was $1.3 \mathrm{~mJ}$ and $2.4 \mathrm{~mJ}$, respectively. The temporal delay between the reference and control beams is adjustable by a translation stage. The two beams were recombined together by a beam splitter. After this beam splitter, both beams were focused by a plano-convex lens with a focal length of $50 \mathrm{~cm}$, generating filaments in air. The terahertz radiations generated by these two beams were collected by a pair of parabolic mirrors and detected by an electro-optic sampling (EOS) device with a

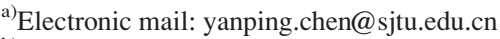

${ }^{\text {b)} E l e c t r o n i c ~ m a i l: ~ z m s h e n g @ s j t u . e d u . c n ~}$
}

$\langle 110\rangle$ oriented ZnTe crystal of $1.5 \mathrm{~mm}$ thickness. ${ }^{9} \mathrm{~A}$ $500-\mu$ m-thick silicon wafer was applied between the two parabolic mirrors to block the residual fundamental pulses and white light from the filaments.

We measured terahertz radiations from filaments generated by the reference and control beams at each specific delay. The terahertz waveforms and their Fourier transform spectra for each temporal delay between the reference and control beams are specified in Figs. 1 and 2, respectively. Figure 1(a) presents the typical terahertz waveform obtained from filaments created by the reference beam only and Fig. 1(b) for the control beam only. Their corresponding spectra are shown in dashed line for the reference beam and dotted line for the control beam in Fig. 2(a). Their spectral profiles over the whole terahertz frequency domain are similar. The spectral amplitude for the reference beam, however, is lower because this beam is weaker compared to the control beam. When both the reference and control beams initiate filaments, the waveforms and spectra of terahertz radiations from the filaments make interesting changes. In the case of no temporal delay between the reference and control beams [Fig. 1(c)], the spectral profiles of terahertz radiation from these two beams are the same as terahertz emission either from the reference beam or from the control beam. And its spectral amplitude is close to the sum of terahertz emission produced by the reference and control beams [solid line in Fig. 2(a)]. When the strong pulse (control beam) is 4-ps delayed from the weak pulse (reference beam), the time difference of the two terahertz pulses generated by these two beams is larger than the terahertz pulses' temporal width [Fig. 1(d)]. Compared with the terahertz waveform obtained with only the control beam in Fig. 1(b), the phase of the terahertz waveform radiated from the delayed control beam slightly changes while the modification in its amplitude is negligible [Fig. 1(d)]. Meanwhile, frequency modulation is observed within the whole terahertz spectral envelope, as shown by solid line in Fig. 2(b). When the reference beam is 

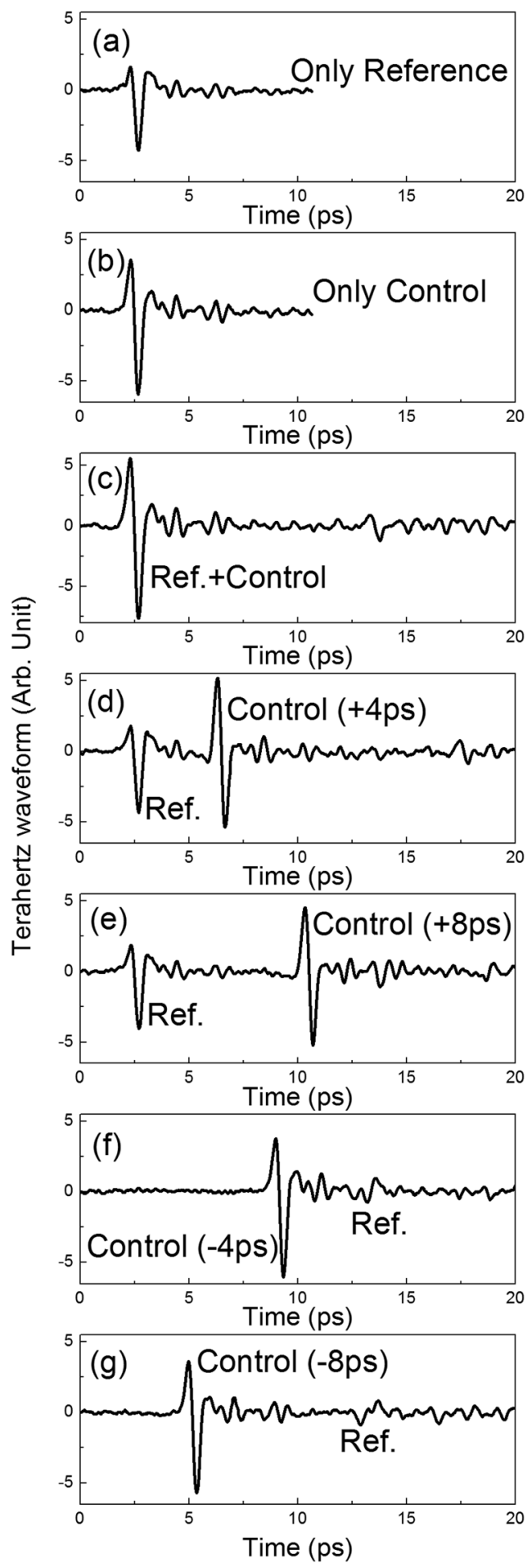

FIG. 1. Waveforms of terahertz radiation from filaments induced by (a) the reference beam, (b) the control beam, (c) the reference beam and control beam simultaneously (same delay), (d) the reference beam and 4-ps delayed control beam, (e) the reference beam and 8-ps delayed control beam, (f) the 4-ps delayed reference beam and control beam, and $(\mathrm{g})$ the 8-ps delayed reference beam and control beam.

8-ps ahead of the control beam, the whole spectrum shows increased frequency modulation accompanied with more periodic peaks and narrower bandwidth, as shown by solid line in Fig. 2(c). The waveform of the terahertz pulse emitting from the 8-ps delayed control beam is quite similar to that obtained with the 4-ps delayed control beam [Figs. 1(d) and $1(\mathrm{e})]$.

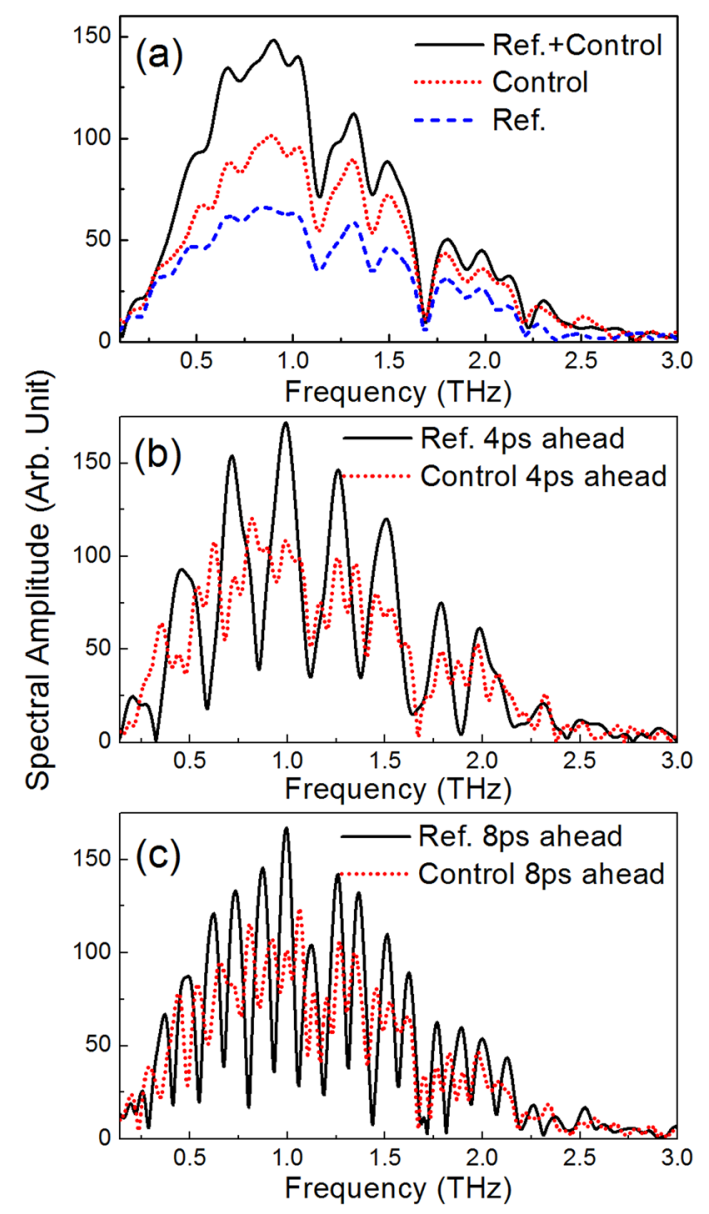

FIG. 2. Spectral amplitudes for terahertz radiation from filaments induced by (a) the reference beam (dashed line), the control beam (dotted line), and both the reference beam and control beam (solid line), (b) the reference beam and 4-ps delayed control beam (solid line), the reference beam, and 4ps ahead control beam (dotted line), and (c) the reference beam and 8-ps delayed control beam (solid line) and the reference beam and 8-ps ahead control beam (dotted line).

When the strong pulse (control beam) propagates ahead of the weak pulse (reference beam), the amplitudes of the terahertz waveforms radiated from the delayed reference beams are reduced to a quarter of the terahertz signal emitting from the original reference beam, as shown in Figs. 1(f) and $1(\mathrm{~g})$. Their corresponding spectral amplitudes are shown in dotted lines in Figs. 2(b) and 2(c). It is obvious that the frequency modulation phenomenon becomes weaker and noisy than the terahertz spectra obtained when the control pulse propagates behind the reference pulse. This dramatic drop in the terahertz amplitude created by the reference pulse is due to the nonlinear interaction between the two filaments generated by the reference and control pulses. When the reference pulse propagates after the control pulse, it will feel the strong plasma defocusing effect induced by filamentation process of the control pulse. Consequently, most of its pulse energy is diffracted out to the energy reservoir, ${ }^{10}$ which results in the generation of a weaker terahertz pulse. However, the energy of the control pulse is high enough to overcome the plasma defocusing effect induced by filamentation process of the reference pulse when the control pulse travels behind the reference pulse. In this situation, therefore, no obvious decrease in the amplitude of the terahertz pulse 
generated by the control beam is observed, except for minor changes in the phase of this pulse.

The evolution of the spectral full width at half maximum of these periodic peaks as a function of the temporal difference between the reference and control pulses is illustrated in Fig. 3. Each point corresponds to an average spectral width of the periodic peaks measured at a specified temporal delay between two pulses in the experiment. All these data are collected while the control beam travels in front of the reference beam. When the temporal separation of the two pulses increases from $1.33 \mathrm{ps}$ to $10.66 \mathrm{ps}$, the terahertz spectral width drops from $0.7 \mathrm{THz}$ to $0.1 \mathrm{THz}$, accordingly. This measurement indicates that the periodic peaks become narrower with the increase of time delay between the two laser pulses. This spectral width curve can be well fitted (solid line, Fig. 3) by using the following equation:

$$
\Delta f \cdot \Delta t=1
$$

where $\Delta f$ is the terahertz spectral width at half maximum and $\Delta t$ is the time difference between the reference and control terahertz pulses. This observation suggests that the terahertz spectral width $(\Delta f)$ only depends on the time difference between two terahertz pulses $(\Delta t)$.

The occurrence of these periodic spectral peaks within the whole terahertz spectral envelope can be explained by interference effects of two delayed terahertz pulses emitting from the filaments formed by two discrete, successive femtosecond laser pulses (a reference pulse and a delayed control pulse). Each spectral peak in the modulated terahertz spectrum represents a constructive interference of this spectral component. The intensity at each frequency is a coherent superposition of the spectral components from the reference and control terahertz pulses at this measured wavelength $(\lambda),{ }^{11}$ i.e.,

$$
I(\lambda) \sim\left[E(\lambda)_{0}^{r e f}+E(\lambda)_{0}^{\text {control }}\right]^{2} \cos ^{2}\left(\frac{\pi d}{\lambda}\right)+C(\lambda),
$$

where $d$ is the distance between the reference and control pulses, $E(\lambda)_{0}^{r e f}$ and $E(\lambda)_{0}^{\text {control }}$ are spectral amplitudes of terahertz components from the reference and control terahertz pulses at wavelength $\lambda$, respectively. Here, $C(\lambda)$ depends on

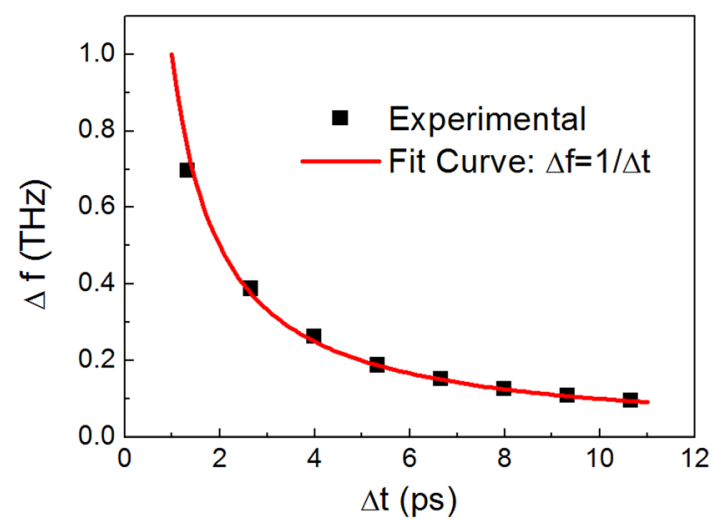

FIG. 3. Spectral width of periodic peaks as a function of the temporal difference between the reference and control pulses. the difference of $E(\lambda)_{0}^{r e f}$ and $E(\lambda)_{0}^{\text {control }}$, which equals to zero when the reference and control beams have the same energy. Figure 4 illustrates the experimental and calculated spectral intensity of two successive terahertz pulses emitting from filaments formed by the reference beam and 4-ps delayed control beam. The experimental curve is obtained by squaring the amplitude of the terahertz spectrum acquired when the control beam propagates 4 ps before the reference beam [solid curve in Fig. 2(b)]. The calculation is based on Eq. (2) and it fits the experimental curve very well. Some minor difference comes from nonlinear interaction of two successive filaments. ${ }^{12-14}$ The plasma induced by the leading reference beam affects the formation of the filaments generated by the trailing control beam, which results in some nonlinear effects superposed on the spectrum of terahertz spectral interference. Equation (2) also indicates that the frequency of spectral modulation is related to the temporal separation between the two successive terahertz pulses. Higher frequency modulation can be obtained when the two terahertz pulses depart further from each other. Therefore, the modulation frequency in the terahertz spectral envelope can be easily manipulated by modification of the temporal separation of the reference and control pulses. Also, the spectral peaks can be smoothly tuned in the same way.

In conclusion, we have produced spectral interference in the terahertz region by two terahertz pulses emitting from filaments induced by two successive femtosecond pulses. The amplitude of the whole terahertz spectral envelope is enhanced by interference of two terahertz pulses. The central frequency and the spectral bandwidth of the terahertz periodic peaks are tunable by manipulating the temporal separation between the leading and trailing terahertz pulses. Not only can this terahertz spectrum could improve the signal to noise ratio in the terahertz time-domain spectroscopy by tuning the spectral peaks to the absorption lines of the interested samples but also provides a unique way to study the nonlinear interaction of time-delayed filaments by

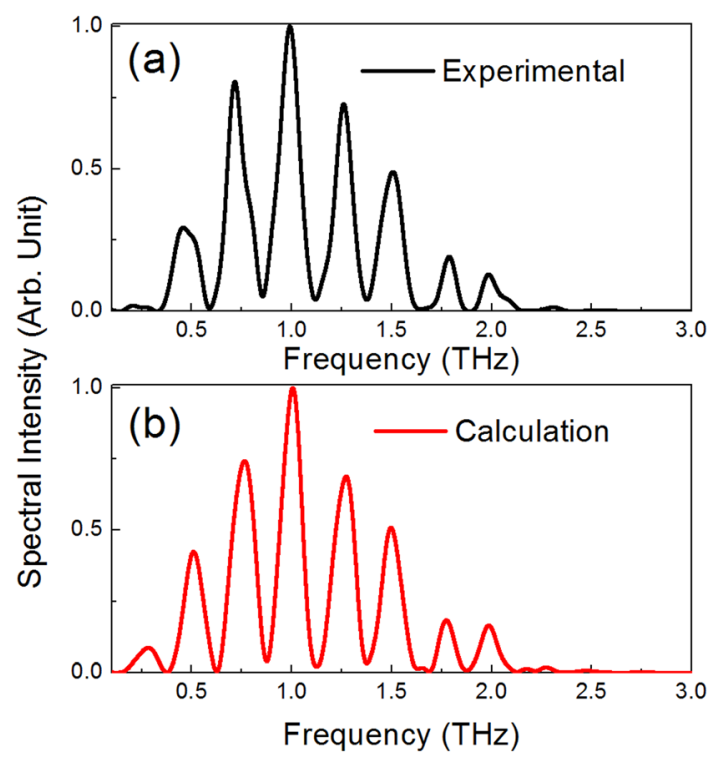

FIG. 4. (a) Experimental and (b) calculated spectral intensity of two successive terahertz pulses emitting from filaments formed by the reference beam and 4-ps delayed control beam. 